\title{
Associations Between Fatty Acids Intake And Tension-Type Headache: A Cross-Sectional Study
}

Diego Domínguez Balmaseda ( $\square$ diego.dominguez@universidadeuropea.es )

European University of Madrid

Guillermo García Pérez de Sevilla

European University of Madrid

Angel González de la Flor

European University of Madrid

José Angel Del Blanco Muñiz

European University of Madrid

\section{Research Article}

Keywords: Tension-type headache, monounsaturated fatty acids (MUFA), polyunsaturated fatty acids (PUFA), Omega-3 ( $\omega-3)$, Omega-6 ( $\omega-6)$.

Posted Date: February 16th, 2022

DOI: https://doi.org/10.21203/rs.3.rs-1350857/v1

License: (c) (1) This work is licensed under a Creative Commons Attribution 4.0 International License. Read Full License 


\section{Abstract}

Introduction: Patients with tension-type headache (TTH) are characterized by recurrent pain that can become disabling, accompanied by sensory, motor and neurovascular changes, with a prevalence of between 36 and $78 \%$ in adults. Identifying the dietary triggers of headaches has led to defining dietary strategies to prevent this disease since excessive consumption, for example, of fats is widely accepted as unhealthy. Within the essential polyunsaturated fatty acids (PUFA), they have shown that Omega-3 fatty acids ( $\omega-3)$ have anti-inflammatory and neuroprotective effects of $\omega-3$ fatty acids. In contrast, excessive dietary intake of Omega- 6 ( $\omega-6)$ fatty acids, or an $\omega-6: \omega 3 \geq 5$ ratio, typical of Western diets, has been associated with a higher prevalence of headaches.

Objectives: The objectives of the present study were to compare dietary fatty acid intake between participants with and without chronic TTH and to investigate the cross-sectional association of dietary fatty acid intake, pain characteristics, and quality of life in patients with chronic TTH.

Methods: An observational study was conducted following the Strengthening Statement of the Reporting Initiative on Observational Studies in Epidemiology (STROBE), comparing healthy participants and participants diagnosed with chronic CT, for more than six months according to the Society's Headache Study Group criteria. Spanish of Neurology. For the study we used characteristics of headache episodes, headache impact test and diet analysis as variables.

Results: There were no significant differences between the TTH group and the CG in anthropometric and demographic variables. The TTH group reported a significantly higher intake of SFA. Furthermore, significant associations were found between PUFA intake and headache characteristics. However, even though the TTH group reported a significant impact of headaches on their activities of daily living according to the HIT-6 questionnaire, there were no significant correlations between the HIT- 6 score and fat intake. Regarding the total daily intake of MUFAs, PUFAs, and $\omega-6$ fatty acids, there were no significant differences between the TTH group and the control group. However, there was a moderate positive correlation between $\omega-6$ fatty acid intake and headache intensity.

Conclusion: Chronic tension-type headache patients showed higher intakes of SFA, but similar intakes of MUFAs, PUFAs and $\omega-6$ : $\omega-3$ ratio when compared to controls. In addition, $\Omega-6$ fatty acids intake and $\omega-6$ : $\omega-3$ ratio were associated with headache episodes.

\section{Introduction}

Tension-type headache (TTH) is characterized by recurrent headaches that can become disabling, often accompanied by sensory, motor and neurovascular alterations, with a prevalence of between 36 and $78 \%$ in adults. $(1,2)$.

Although the cause of TTH is unclear and multifactorial, the pathophysiology of migraine involves immune response factors and oxidative stress $(3,4)$, which could lead to neuronal inflammation $(2,5)$. 
Though, the pathogenesis and progression of TTH appears to be influenced by a combination of physiological, psychosocial, and lifestyle factors $(6,7)$. Some of these factors, including nutritional patterns, are amenable to behavioral and clinical interventions (8). Recently, TTH has been associated with dietary elements (9). In fact, advances in understanding the mechanisms underlying headache pathogenesis and how dietary factors may interfere with those mechanisms have encouraged investigators to consider diet as a disease-modifying agent (10).

Identifying the dietary triggers of headaches has led to defining dietary strategies to prevent this disease (11). For example, a higher intake of alcohol or canned foods rich in nitrates and nitrites, monosodium glutamate and artificial sweeteners, has been linked to a higher prevalence of headache (12). In addition, excess fat intake is widely accepted as unhealthy, but the type of fat intake is more clinically relevant (13).

Fatty acids can be classified depending on their length and degree of saturation into saturated fatty acids (SFAs), monounsaturated fatty acids (MUFAs) and polyunsaturated fatty acids (PUFAs). Among essential PUFAs, Omega-3 ( $\omega-3)$ fatty acids such as eicosatetraenoic acid (EPA) and docosahexaenoic acid (DHA) are crucial for the nerve system's functioning. Several studies have shown the effects of antiinflammatory and neuroprotective effects of the $\omega-3$ fatty acids $(14,15)$. On the contrary, excess of Omega- 6 ( $\omega-6)$ fatty acids dietary intake, or an $\omega-6$ : $\omega 3$ ratio $\geq 5$, which is typical in western diets, has been associated with a higher prevalence of headaches $(10,16)$. In addition, a higher dietary intake of $\omega-3$ can reduce the frequency of headaches, according to a recent study (17). Similarly, excess SFAs intake could favor inflammatory reactions in some tissues and could be responsible for causing headaches (18).

Therefore, the diet must be considered as a relevant modifiable factor that needs more attention. Given the paucity of research about this topic and gaps in knowledge, the aims of the present study were: (i) to compare the dietary intake of fatty acids between participants with and without chronic TTH; (ii) to investigate the cross-sectional association of fatty acids dietary intake, pain characteristics and quality of life in patients with chronic TTH.

\section{Methods}

\section{Study design}

An observational study following the Strengthening the Reporting of Observational Studies in Epidemiology (STROBE) Initiative Statement (19) was conducted, comparing healthy participants and participants with diagnosed TTH for more than six months according to the criteria of the Headache Study Group of the Spanish Society of Neurology (20).

The study protocol adhered to the principles of the 1964 Declaration of Helsinki and its subsequent clarifications and was approved by the Research Ethics Committee of the Rey Juan Carlos University of Madrid (reference number: 1802202105721). 


\section{Participants}

Recruitment of participants was carried out among students and workers at the European University of Madrid. The participants previously went to their medical center, where they were diagnosed with chronic TTH by their neurologist, following the criteria of the International Headache Society (IHS) classification of headaches, in its third edition. The diagnostic criteria followed were Headache that occurs on average more than 15 days per month for more than 3 months (more than 180 days per year) with a duration of minutes to days, or without remission. With a bilateral or oppressive quality pain, of mild or moderate intensity that could present with mild nausea. Participants were included in the study when they fulfilled the following criteria: 1) Adults aged 18-65 years; 2 ) for the TTH group (TTHG) only: having TTH for more than six months (diagnosed with chronic TTH by their neurologist, following the criteria of the International Headache Society classification of headaches, in its third edition) (21).

\section{Variables}

Anthropometric variables were height in centimeters $(\mathrm{cm})$, and weight in kilograms $(\mathrm{kg})$. Height was measured with a measuring rod (Ano Sayol SL, Barcelona, Spain) and weight with a mechanical scale (Asimed T2, Barcelona, Spain). Body mass index (BMI) was calculated as Weight (kg) / height (m2) following Shephard's protocol (22).

- Characteristics of headache episodes:

The protocol of Gago-Veiga et al. was followed to measure the duration of the headaches expressed in hours, the intensity of the tension headache on the numeric pain rating scale from 0 to 10 and the frequency in days of the episodes of the subject (20).

\section{- Headache Impact Test}

The Headache Impact Test (HIT-6) measures the impact that headaches have on daily activity tasks. Regarding the severity of the impact, $\geq 60$ means very severe impact; 56-59 significant impact; 50-55 moderate impact; $\leq 49$ little impact (23).

\section{- Diet Analysis}

The diet of the participants was analyzed through the use of the validated Food Frequency Questionnaires (CFCA) (24). Data were analyzed using Dietsource 3.0 software (Novartis, Barcelona, Spain) to obtain dietary intake of SFAs (g), MUFAs (g), PUFAs (g), $\omega-6$ fatty acids (g), and $\omega-3$ fatty acids $(\mathrm{g})$, as well as the total energy intake $(\mathrm{kcal})$. Then, the $\omega-6: \omega-3$ ratio was calculated using the total intake values of $\omega-6$ and $\omega-3$ (25).

Statistical analysis

A descriptive analysis was developed for all the participants using mean \pm standard deviation (SD). Then, Shapiro-Wilk test was employed to assess the normality of the variables (26). For non-parametric 
variables, the Mann-Whitney U test was conducted, while the independent samples t-test was employed to identify significant differences between groups. In addition, to analyze the relationship between continuous variables, Spearman correlation test and Pearson correlation test were performed for the nonparametric and the parametric variables, respectively. The magnitudes of correlation between continuous variables were qualitatively interpreted using the following criteria: trivial $(r \leq 0.1)$, small $(r=0.1-0.3)$, moderate $(r=0.3-0.5)$, large $(r=0.5-0.7)$, very large $(r=0.7-0.9)$ and almost perfect $(r \geq 0.9)(27)$.

The statistical significance was set at an alpha level of $<0.05$. All analyses were conducted using IBM SPSS for Windows (version 25, IBM Corporation, Armonk, New York).

\section{Results}

\section{Characteristics of the sample}

There were no significant differences between the TTHG and the CG regarding the age of the participants (37.58 \pm 13.36 versus $42.57 \pm 8.11$ years; $p=0.12)$, BMI ( $25.18 \pm 4.62$ versus $\left.24.99 \pm 3.95 \mathrm{~kg} / \mathrm{m}^{2} ; p=0.88\right)$, and nutritional energy intake $(2175.08 \pm 519.58$ versus $1960.48 \pm 403.16 \mathrm{kcal} ; \mathrm{p}=0.11)$.

\section{Headache's episodes}

For the TTHG, the mean HIT- 6 score was $60.51 \pm 5.84$, the mean headache intensity was $7.15 \pm 1.32$, headache duration $11.00 \pm 5.56$ hours, and headache frequency $11.71 \pm 9.77$ days a month.

\section{Fat intake}

The TTHG had a significantly higher intake of saturated fat compared to the CG (34.40 \pm 12.73 versus $20.74 \pm 6.18 \mathrm{~g} ; \mathrm{p}<0.01)$. There were no differences between groups regarding the intake of MUFA (43.58 \pm 17.65 versus $40.55 \pm 13.99 \mathrm{~g} ; \mathrm{p}=0.51)$, PUFA $(10.27 \pm 3.21$ versus $9.79 \pm 6.42 \mathrm{~g} ; \mathrm{p}=0.15)$, Omega- 6 fatty acids $(6.86 \pm 2.46$ versus $6.56 \pm 2.98 \mathrm{~g} ; \mathrm{p}=0.44)$, Omega- 3 fatty acids $(1.17 \pm 0.39$ versus $1.13 \pm 0.75 \mathrm{~g}$; $p=0.20)$, and Omega 6: Omega 3 ratio $(6.33 \pm 2.67$ versus $7.08 \pm 2.97 ; p=0.33)$.

Table I Comparative analysis of nutrients daily intake between tension-type headache group $(n=24)$ and control group $(\mathrm{n}=24)$ 


\begin{tabular}{|llll|}
\hline Variables & $\begin{array}{l}\text { Tension-type headache group Mean } \pm \\
\text { SD }\end{array}$ & $\begin{array}{l}\text { Control Group } \\
\text { Mean } \pm \text { SD }\end{array}$ & p-value \\
\hline Age (years) & $37.58 \pm 13.36$ & $42.57 \pm 8.11$ & 0.12 \\
\hline $\begin{array}{l}\text { Body Mass Index } \\
\left(\mathrm{kg} / \mathrm{m}^{2}\right)\end{array}$ & $25.18 \pm 4.62$ & $24.99 \pm 3.95$ & 0.88 \\
\hline Kcal & $2175.08 \pm 519.58$ & $1960.48 \pm 403.16$ & 0.11 \\
\hline SFA $(\mathrm{g})$ & $34.40 \pm 12.73$ & $20.74 \pm 6.18$ & $<0.01^{\star}$ \\
\hline MUFA (g) & $43.58 \pm 17.65$ & $40.55 \pm 13.99$ & 0.51 \\
\hline PUFA (g) & $10.27 \pm 3.21$ & $9.79 \pm 6.42$ & 0.15 \\
\hline$\omega-6$ fatty acids $(\mathrm{g})$ & $6.86 \pm 2.46$ & $6.56 \pm 2.98$ & 0.44 \\
\hline$\omega-3$ fatty acids $(\mathrm{g})$ & $1.17 \pm 0.39$ & $1.13 \pm 0.75$ & 0.20 \\
\hline$\omega-6: \omega$-3 ratio & $6.33 \pm 2.67$ & $7.08 \pm 2.97$ & 0.33 \\
\hline
\end{tabular}

Abbreviations: saturated fatty acid, SFA; monounsaturated fatty acid, MUFA; polyunsaturated fatty acid, PUFA. P-value was calculated with the independent samples t-test for parametric variables, and the Mann-Whitney $U$ test for the non-parametric variables. ${ }^{*} p<0.05$ (t-test); $\otimes p<0.05$ (Mann-Whitney U test).

\section{Correlations between the continuous variables}

$\Omega-6$ fatty acids intake showed a positive moderate correlation with headache intensity $(r=0.48 ; p=0.02)$.

In turn, $\omega-6: \omega-3$ ratio showed a negative moderate correlation with headache duration $(r=-0.41 ; p=0.04)$ and frequency $(r=-0.42 ; p=0.03)$.

No other significant correlations were found.

\section{Discussion}

This novel research provides information about dietary habits in TTH patients, with special attention to the intake fatty acids. The results were compared to the dietary habits of healthy patients without TTH participants who had a similar daily caloric intake. The TTH group reported significant higher SFAs intake. In addition, significant associations were found between PUFAs intake and the headaches characteristics.

The participants of this study were middle-aged, slightly overweight, who reported a normal caloric intake of $2000 \mathrm{kcal}$ per day. With respect to SFAs, the TTH group reported a significant higher daily intake compared to the control group. In addition, the SFAs intake of the TTH was $34.40 \mathrm{~g}$, equivalent to 310 $\mathrm{kcal}$, or $14 \%$ of daily caloric intake, exceeding the health recommendations(28). SFAs are mainly found in 
butter and red, processed meats (29). According to Fritsche et al., excess SFAs intake could favor inflammatory reactions and could be responsible for causing headache (18). In a recent study, neuroinflammation was implicated in the pathogenesis of headache, as increased levels of proinflammatory cytokines, such as interleukin-1 $\beta$ and tumor necrosis factor-a are involved in the immune responses associated with headache (30). These findings have been reported in several studies (17,31-34). However, even though the TTH reported a significant impact of headaches on their daily living activities according to the HIT-6 questionnaire, there were no significant correlations between the HIT-6 score and fat intake.

Regarding the total daily intake of MUFAs, PUFAs, and $\omega-6$ fatty acids, there were no significant differences between the TTH group and the control group. However, there was a positive moderate correlation between $\omega-6$ fatty acids intake and headache intensity. In this line, dietary patterns with high consumption of $\omega-6$ have been associated with an increased risk of suffering from diseases associated with inflammatory processes $(14,18,35)$ and a higher prevalence of migraine headaches $(10,16,17)$. A possible explanation could be that the most common $\omega-6$ fatty acid, the linoleic acid, can give rise to arachidonic acid, which has inflammatory effects. Foods rich in $\omega-6$ are oils derived from seeds (sunflower, corn, sesame), nuts (walnuts, pine nuts, peanuts, almonds, hazelnuts and pistachios), quinoa, whole grains, meats (especially chicken and turkey), sausages and eggs $(14,18,36)$.

The TTH group and the control group showed similar intakes of $\omega-3$ fatty acids, and this variable did not show any significant correlation with the headache's episodes. However, the $\omega 6: \omega-3$ ratio is more clinically relevant, because if in the cell membranes the presence of $\omega-6$ is well above the $\omega-3$ fatty acids there may be an inflammatory response. In fact, it is desirable that $\omega-6$ and $\omega-3$ fatty acids be consumed in similar amounts (ratio 1:1), although this is very rare. In the usual diet in the United States, this ratio is 20:1. Most experts recommend not exceeding a 5:1 ratio, because in that case, $\omega-6$ fatty acids produce an inflammatory effect that can promote headaches, among other detrimental effects $(10,37)$. In the present study, in both groups the $\omega 6: \omega-3$ ratio was higher than $5: 1$, without significant differences between groups. Additionally, there were moderate negative correlations between this fatty acids ratio and the duration $(r=-0.41 ; p=0.04)$ and frequency $(r=-0.42 ; p=0.03)$ of headaches episodes. In this line, in a recent study the incidence of headaches was lower in participants who increased the $\omega-3$ fatty acids intake and simultaneously reduced the intake of $\omega-6$ linoleic acid (17). This effect could be explained because $\omega-6$ fatty acids synthesize inflammatory hormones and because the excess of $\omega-6$ prevents $\omega-3$ fatty acids from fulfilling their functions (38).

Therefore, the dietary advice is to increase $\omega-3$ fatty acids intake, which are often lacking in the diet of the western population, and to reduce $\omega-6$ intake, which are often consumed in excess. $\Omega-3$ fatty acids are abundant in oily fish, seafood, seeds, and nuts (29). Regarding oily fish, sardines, mackerel, herring or anchovies are more suitable than salmon, swordfish or tuna, because larger species are more contaminated with mercury (39). 
This novel study provides useful information for health practitioners regarding the management of patients with TTH. An excess of SFAs intake and a $\omega-6: \omega 3$ ratio higher than 5:1 could have a negative impact on the duration, frequency and intensity of headaches episodes. Therefore, the diet should be assessed in patients with TTH.

Future studies should confirm these findings by carrying out dietary interventions in TTH patients, aiming to increase the intake of $\omega-3$ fatty acids. Also, these studies should measure inflammatory markers and analyze possible associations with intensity, duration, and frequency of headaches episodes.

\section{Limitations of the study}

The results of this study should be considered in light of the following limitations: (i) The observational nature of this study does not allow causation; (ii) All data were self-reported, about dietary intake, and may be subject to information bias.

\section{Conclusions}

Chronic tension-type headache patients showed higher intakes of SFA, but similar intakes of MUFAs, PUFAs and $\omega-6: \omega-3$ ratio when compared to controls. In addition, $\Omega-6$ fatty acids intake and $\omega-6: \omega-3$ ratio were associated with headache episodes.

\section{Declarations}

\section{FUNDING}

There was no funding.

\section{CONFLICT OF INTEREST}

There was no conflict of interest.

\section{References}

1. Ferrante T, Manzoni GC, Russo M, Camarda C, Taga A, Veronesi L, et al. Prevalence of tension-type headache in adult general population: the PACE study and review of the literature. Neurol Sci [Internet]. 2013 [cited 2022 Jan 25];34 Suppl 1(SUPPL. 1). Available from:

https://pubmed.ncbi.nlm.nih.gov/23695063/

2. Manzoni GC, Stovner LJ. Epidemiology of headache. Handb Clin Neurol. 2010;97:3-22.

3. Kowalska M, Prendecki M, Kozubski W, Lianeri M, Dorszewska J. Molecular factors in migraine. Oncotarget [Internet]. 2016 [cited 2022 Feb 8];7(31):50708. Available from: /pmc/articles/PMC5226615/ 
4. Borkum JM. Migraine Triggers and Oxidative Stress: A Narrative Review and Synthesis. Headache [Internet]. 2016 Jan 1 [cited 2022 Feb 8];56(1):12-35. Available from:

https://pubmed.ncbi.nlm.nih.gov/26639834/

5. Bigal ME, Lipton RB, Holland PR, Goadsby PJ. Obesity, migraine, and chronic migraine: possible mechanisms of interaction. Neurology [Internet]. 2007 [cited 2022 Feb 8];68(21):1851-61. Available from: https://pubmed.ncbi.nlm.nih.gov/17515549/

6. Dale PC, Thomas JC, Hazle CR. Physical therapist clinical reasoning and classification inconsistencies in headache disorders: a United States survey. J Man Manip Ther [Internet]. 2020 Jan 1 [cited 2022 Jan 25];28(1):28-40. Available from: https://pubmed.ncbi.nlm.nih.gov/31373539/

7. Crystal SC, Robbins MS. Epidemiology of tension-type headache. Curr Pain Headache Rep. 2010 Dec;14(6):449-54.

8. Mills SEE, Nicolson KP, Smith BH. Chronic pain: a review of its epidemiology and associated factors in population-based studies. Br J Anaesth. 2019 Aug;123(2):e273-83.

9. Sanders AE, Shaikh SR, Slade GD. Long-chain omega-3 fatty acids and headache in the U.S. population. Prostaglandins Leukot Essent Fatty Acids [Internet]. 2018 Aug 1 [cited 2022 Jan 25];135:4753. Available from: https://pubmed.ncbi.nlm.nih.gov/30103932/

10. Ramsden CE, Faurot KR, Zamora D, Palsson OS, MacIntosh BA, Gaylord S, et al. Targeted alterations in dietary n-3 and $n-6$ fatty acids improve life functioning and reduce psychological distress among patients with chronic headache: a secondary analysis of a randomized trial. Pain. 2015 Apr;156(4):58796.

11. Gazerani P. Migraine and Diet. Nutrients [Internet]. 2020 Jun 1 [cited 2022 Jan 25];12(6):1-11. Available from: https://pubmed.ncbi.nlm.nih.gov/32503158/

12. Zaeem Z, Zhou L, Dilli E. Headaches: a Review of the Role of Dietary Factors. Curr Neurol Neurosci Rep [Internet]. 2016 Nov 1 [cited 2022 Feb 8];16(11). Available from: https://pubmed.ncbi.nlm.nih.gov/27714637/

13. Bagga D, Wang L, Farias-Eisner R, Glaspy JA, Reddy ST. Differential effects of prostaglandin derived from omega- 6 and omega-3 polyunsaturated fatty acids on COX-2 expression and IL- 6 secretion. Proc Natl Acad Sci U S A. 2003 Feb;100(4):1751-6.

14. De Caterina R, Madonna R, Massaro M. Effects of omega-3 fatty acids on cytokines and adhesion molecules. Curr Atheroscler Rep. 2004 Nov;6(6):485-91.

15. Forman MS, Lal D, Zhang B, Dabir D V, Swanson E, Lee VM-Y, et al. Transgenic mouse model of tau pathology in astrocytes leading to nervous system degeneration. J Neurosci. 2005 Apr;25(14):3539-50. 
16. Razeghi Jahromi S, Ghorbani Z, Martelletti P, Lampl C, Togha M. Association of diet and headache. J Headache Pain. 2019 Nov;20(1):106.

17. Ramsden CE, Zamora D, Faurot KR, MacIntosh B, Horowitz M, Keyes GS, et al. Dietary alteration of n3 and $n-6$ fatty acids for headache reduction in adults with migraine: randomized controlled trial. BMJ. 2021 Jun;374:n1448.

18. Fritsche KL. The science of fatty acids and inflammation. Adv Nutr [Internet]. 2015 [cited 2022 Jan 25];6(3):293S-301S. Available from: https://pubmed.ncbi.nlm.nih.gov/25979502/

19. Elm E von, Altman DG, Egger M, Pocock SJ, Gøtzsche PC, Vandenbroucke JP, et al. The Strengthening the Reporting of Observational Studies in Epidemiology (STROBE) Statement: Guidelines for Reporting Observational Studies. PLoS Med. 2007;4(10):1623-7.

20. Gago-Veiga AB, Camiña Muñiz J, García-Azorín D, González-Quintanilla V, Ordás CM, Torres-Ferrus M, et al. Headache: What to ask, how to examine, and what scales to use. Recommendations of the Spanish Society of Neurology's Headache Study Group. Neurologia. Spanish Society of Neurology; 2019.

21. Olesen J. Headache Classification Committee of the International Headache Society (IHS) The International Classification of Headache Disorders. 3rd ed. Vol. 38. Cephalalgia. SAGE Publications Ltd; $1-211 \mathrm{p}$.

22. Shephard RJ. Qualified fitness and exercise as professionals and exercise prescription: Evolution of the PAR-Q and Canadian Aerobic Fitness Test. J Phys Act Heal. 2015 Apr 1;12(4):454-61.

23. Kosinski M, Bayliss MS, Bjorner JB, Ware JE, Garber WH, Batenhorst A, et al. A six-item short-form survey for measuring headache impact: the HIT-6. Qual Life Res [Internet]. 2003 Dec [cited 2021 Nov 22];12(8):963-74. Available from: https://pubmed.ncbi.nlm.nih.gov/14651415/

24. Goni Mateos L, Aray Miranda M, Martínez AH, Cuervo Zapatel M. Validation of a food groups frequency questionnaire based in an exchange system. Nutr Hosp [Internet]. 2016 [cited 2021 Apr 26];33(6):1391-9. Available from: http://dx.doi.org/10.20960/nh.800

25. Simopoulos AP. The importance of the ratio of omega-6/omega-3 essential fatty acids. Biomed Pharmacother. 2002 Oct;56(8):365-79.

26. Ghasemi A, Zahediasl S. Normality tests for statistical analysis: A guide for non-statisticians. Int J Endocrinol Metab. 2012;10(2):486-9.

27. Hopkins WG, Marshall SW, Batterham AM, Hanin J. Progressive Statistics for Studies in Sports Medicine and Exercise Science. Med Sci Sports Exerc. 2009;41(1):3-12.

28. Harcombe Z. US dietary guidelines: is saturated fat a nutrient of concern? $\mathrm{Br} \mathrm{J}$ Sports Med. 2019;53(22):1393-6. 
29. García Pérez de Sevilla G, Sánchez-Pinto Pinto B. Effectiveness of Workplace Mediterranean Diet Interventions on Cardiometabolic Risk Factors: A Systematic Review: Workplace Health Saf. 2022;

30. Yamanaka G, Suzuki S, Morishita N, Takeshita M, Kanou K, Takamatsu T, et al. Role of neuroinflammation and blood-brain barrier permutability on migraine. Int J Mol Sci. 2021 Aug 2;22(16).

31. Domínguez-Vivero C, Leira Y, López-Ferreiro A, Saavedra M, Rodríguez-Osorio X, Sobrino T, et al. Pentraxin 3 (PTX3): A Molecular Marker of Endothelial Dysfunction in Chronic Migraine. J Clin Med [Internet]. 2020 Mar 1 [cited 2022 Feb 8];9(3). Available from: /pmc/articles/PMC7141491/

32. Durham P, Papapetropoulos S. Biomarkers associated with migraine and their potential role in migraine management. Headache. 2013 Sep;53(8):1262-77.

33. Avona A, Price TJ, Dussor G. Interleukin-6 induces spatially dependent whole-body hypersensitivity in rats: implications for extracephalic hypersensitivity in migraine. J Headache Pain. 2021 Dec 1;22(1).

34. Sadeghi O, Maghsoudi Z, Khorvash F, Ghiasvand R, Askari G. The relationship between different fatty acids intake and frequency of migraine attacks. Iran J Nurs Midwifery Res [Internet]. 2015 [cited 2022 Jan 25];20(3):334. Available from: /pmc/articles/PMC4462058/

35. Patterson WL, Georgel PT. Breaking the cycle: The role of omega-3 polyunsaturated fatty acids in inflammation-driven cancers1. Biochem Cell Biol. 2014 Jul 8;92(5):321-8.

36. Ochi E, Tsuchiya Y. Eicosapentaenoic Acid (EPA) and Docosahexaenoic Acid (DHA) in Muscle Damage and Function. Nutrients [Internet]. 2018 Apr 29 [cited 2019 May 17];10(5):552. Available from: http://www.mdpi.com/2072-6643/10/5/552

37. Ramsden CE, Faurot KR, Zamora D, Suchindran CM, Macintosh BA, Gaylord S, et al. Targeted alteration of dietary n-3 and n-6 fatty acids for the treatment of chronic headaches: a randomized trial. Pain [Internet]. 2013 [cited 2022 Feb 8];154(11):2441-51. Available from: https://pubmed.ncbi.nlm.nih.gov/23886520/

38. Boyd JT, LoCoco PM, Furr AR, Bendele MR, Tram M, Li Q, et al. Elevated dietary $\omega-6$ polyunsaturated fatty acids induce reversible peripheral nerve dysfunction that exacerbates comorbid pain conditions. Nat Metab [Internet]. 2021 Jun 1 [cited 2022 Feb 8];3(6):762-73. Available from: https://pubmed.ncbi.nlm.nih.gov/34140694/

39. Claudia Torrejón D. Mercurio y Salud. Rev Med Chile. 2014;142:1174-80. 